
\title{
Resesarch Sulure \\ Computational Methods for Predicting the Outcome of Thoracic Transplantation
}

Raji C.G ( $\square$ rajicg80@gmail.com )

MEA Engineering College https://orcid.org/0000-0003-0896-5184

Safna A.K

MEA Engineering College

\section{Research}

Keywords: Thoracic transplantation, Multi layer perceptron, Radial basis function, Validation, Survival prediction.

Posted Date: January 27th, 2022

DOI: https://doi.org/10.21203/rs.3.rs-1162094/v1

License: (1) This work is licensed under a Creative Commons Attribution 4.0 International License. Read Full License 


\title{
Computational Methods for Predicting the Outcome of Thoracic Transplantation
}

\author{
Raji C. $\mathrm{G}^{1, *}$ Safna A. $\mathrm{K}^{2}$ \\ ${ }^{1, *}, 2$ Computer Science and Engineering, MEA Engineering College, \\ Perinthalmanna, Kerala, India, \\ E-mail: rajicg80@gmail.com,safnaanikkadan@gmail.com \\ ORCID id: 0000-0003-0896-5184
}

\begin{abstract}
Cardiac disease and the death rates due to coronary heart failure and cardiomyopathy are increasing. Thoracic transplantation is now a widely accepted therapeutic option for end-stage cardiac failure. The survival rate after the organ transplantation is crucial. Survival prediction after heart transplantation is a hot area of research. The use of conventional statistical techniques is computationally expensive and does not provide reliable solutions. Artificial Neural Networks based survival prediction helps surgeons make precise decisions and predict the best outcomes. The proposed system implements multi-layer perceptron algorithm, which shows good performance in survival prediction. We also implemented our work in the Radial Basis Function Network model to prove the accuracy of proposed model. For this research study, data were collected from United Network for Organ Sharing database and extracted the relevant thoracic transplantation survival prediction attributes with the help of suitable data mining techniques. We obtained an accuracy of $97.1 \%$ from the multi-layer perceptron model with the evaluation of various performance measures. In order to assure the validity of the proposed model we implemented the Radial Basis Function model and obtained an accuracy of $92.37 \%$. We collated the accuracy of proposed survival prediction models with existing systems and proved that the proposed system appeared to be best for survival prediction with higher accuracy compared to $85.9 \%$ in the existing system. The outcome of the model will be an asset for the lifesaving procedures in the medical field.
\end{abstract}

Keywords: Thoracic transplantation, Multi layer perceptron, Radial basis function, Validation, Survival prediction.

\section{Introduction}

Thoracic transplantation (TT) is a surgical procedure that can be considered as the only medical procedure suitable for end-stage cardiac disease. Thoracic transplants are performed when other medical treatments for cardiac problems have not worked, resulting in coronary failure. In adults, cardiac infarction can be generated by weakening of the cardiac muscle (cardiomyopathy), advanced heart failure, arrhythmia, inherited heart disease, coronary thrombosis, and heart valve diseases [1]. Doctors consider many factors when evaluating patients for transplant, including analysis of liver and kidney function tests to work out whether poor blood 
flow is hampering the vital functions of those organs [1]. The surgical outcome of TT is dependent on many aspects, such as severity of cardiac illness, age, and health condition for the pumping of blood [1]. The survival prediction of thoracic transplantation is an extensive area of research. In the clinical research, the survival prediction is done by implementing many mathematical and statistical probability models. In clinical outcomes, the survival prediction is done by using Wilcoxon rank sum and chi-square tests and subjective comparisons. In the computational methods, the outcome is predicted by using different machine learning techniques. The survival predictions are done by using different machine learning algorithms like linear regression, logistic regression, K-Nearest Neighbor, Support Vector Machine, and Naive Bayes. The proposed system implements accurate survival prediction by using Artificial Neural Networks (ANN).

ANNs are models that are drafted to resolve problems by trying to mimic the configuration and activity of our nervous system [2]. Neural networks are supported simulated neurons that are linked together in a specific pattern to form networks [2]. A neural network relates the human brain in two ways. A neural network gathers knowledge through learning. Neural network knowledge is reposited with in the link strengths known as synaptic weight.

\section{Related Research}

In 2007, Davies et al. proposed a new method to evaluate early post-transplant thoracic survival in high-risk pediatric patients [3]. They collected the data from the United Network for Organ Sharing (UNOS) thoracic registry and implemented the survival prediction by using statistical probability method [3]. In 2009, Asil et al. introduced a method for predicting graft survival for heart-lung transplantation patients by using different data mining methods [4]. They implemented the graft survival prediction by using machine learning techniques, such as decision trees, logistic regression and multilayer perceptron on a large feature rich dataset [4]. The survival prediction obtained an accuracy of only $85.9 \%$ [4]. In 2012, Weiss et al. developed a significant donor risk index to prognosticate short term mortality in orthotropic thoracic transplantation [5], [6]. They utilized the UNOS STAR file to develop a significant donor risk score for orthotropic heart transplantation. They created and validated donor-based risk score elements such as ischemic time, age of donor, mismatching of race, blood urea nitrogen, and creatinine ratio and implemented them by using a logistic regression model [5], [6]. In this study, the system conducted a retrospective study from administrative dataset and could not conduct scientific validation [5], [6]. In 2012, Chokshi implemented a study on hepatic dysfunction accompanied with impaired clinical outcome after thoracic transplantation. They calculated the model for end-stage liver disease (MELD) and albumin replacing modified MELD after transplantation [7]. The study was conducted by statistical computation method and also the study was in retrospective nature [7]. Later in 2016, Medved et al. introduced a study on an ideal feature set opting to forecast thoracic transplantation outcome for one, five and ten years which was implemented by logistic regression with greedy forward and backward search [8]. As a technique to prognosticate survival, they made use of the Index for Mortality Prediction After Cardiac Transplantation (IMPACT) and the International Heart Transplant Survival Algorithm (IHTSA) [8]. However, the IMPACT could not accurately predict survival for one, five and ten years [8]. In 2016, Ali Dag et al. implemented the prediction 
of graft survival after cardiac transplantation through development of data analytical models [9]. The main aim of the research was to forecast the one, five, and nine year graft survival of patients undergoing a thoracic transplantation surgery via the sequence of analytical models that were dependent on four powerful classification algorithms (for example support vector machines, decision trees, logistic regression and artificial neural networks) [9]. The results showed that logistic regression and neural network models yielded higher performance in the survival prediction with an accuracy of $82.4 \%$ and $81.9 \%$, respectively [9]. Later in 2017, Deshpande et al. introduced a method for automatic prediction of heart rejection using image processing and machine learning techniques [10]. The dataset consisted of whole slide images collected from Children's Healthcare of Atlanta [10]. The classification of the images was done by deep neural networks with regularization and dropout. The proposed system was not capable of performing multiclass classification [10]. In 2018, Medved et al. implemented the result of heart allocation policies using a discrete event model and a neural network algorithm by simulation [11]. Data were collected from the UNOS database for their study [11]. They predicted the status of the patient in the queue and selected the best allocation policy [11].

A number of limitations have been found in various studies to forecast the survival of Thoracic transplantation. Due to the lack of obtaining high accuracy computational models and relevant datasets, the researchers were unable to obtain accurate and precise survival predictions after thoracic transplantation. We used ANN models such as multi-layer perceptron and Radial Basis Function Network models for the survival prediction with the help of the UNOS dataset and performed a successful prediction.

\section{Materials and Methods}

\subsection{Description of Dataset}

The proposed system uses the dataset that was gathered from the UNOS database, which is a non-taxable, pragmatic, curative and pedagogic organization. It is the only one frame of organ procurement and transplant network (OPTN) that is an executive United States (US) government network run by the Health Resources and Services Administration, U.S. Department of Health and Human Services. The UNOS data file consists of multiple pre and post-transplant multiple organ data. From the extensive data file, we obtained files linked to Thoracic transplantation that consisted of five-hundred forty-three attributes and 1,48,509 records.

By recognizing and studying identical relationships between donor and recipient, the Thoracic transplantation survival prediction was examined. Although we had a large dataset, only a small set of relevant data file were used for the prognosis of graft survival. Depending upon the variable GRF_STAT which was designated as nominal, we could evaluate the survival prediction of Thoracic transplantation data. After Thoracic transplantation, cases in which the graft failed are depicted as GRF_STATUS=N and GRF_STATUS=Y shows the success of graft after thoracic transplantation. The age of the patient in the dataset is more important when he or she is going for Thoracic transplantation. The age of donor, AGE_DON and the recipient age, INIT_AGE were represented as numerical years. ABO is the blood group of the recipient and ABO_MAT is the donor recipient's match level which was numerically represented. The mean and standard 
deviation for ABO_MATCH were 1.105 and 0.307 respectively. TOT_SERUM_ALBUM is the recipient total serum albumin in which the normal albumin range was 3.4 to $5.4 \mathrm{~g} / \mathrm{dL}$. The mean and standard deviation for TOT_SERUM_ALBUM were 3.919 and 0.517 respectively. ECMO_TRR provided the recipient status of life support and CREAT_TRR was the recipient serum creatinine at the time of transplantation. Normal blood levels of creatinine range from approximately 0.6 to $1.2 \mathrm{mg} / \mathrm{dL}$. The mean and standard deviation obtained for CREAT_TRR were 0.9 and 0.784 respectively. GTIME is the graft life span days from transplant to the last follow-up which were numerically represented. The gender of the recipient, GENDER was represented as nominal. Whether the patient was resistant to bacterial infection or not was represented by the attribute, RESIST_INF. The need for life support was shown as ECMO_TRR. Both best and baseline recent hemodynamic mean values were collected between 04/01/1994 and $10 / 25 / 1999$ and later the value on 10/25/1999 was selected as the best value. During transplant, whether the patient required life support was indicated by the important attribute, IABP_TRR. ACUTE_REJ_EPI was represented as numeric and was considered a recipient attribute. The lifespan of the graft from transplantation to failure/death/follow-up was represented as GTIME. The attributes, TR_TREJ1Y and PRAMR_CL2 were represented as nominal and numeric respectively. The ISCHITIME attribute was represented as numerical hours for the total ischemic time which was represented. Failure of the graft was represented by the attribute, GRF_FAIL_CAUSE. The DOPAMINE_DON_OLD and INOTROP_AGENTS attributes were represented as nominal donor attributes. The HLA Mismatch level at transplantation was represented by HLAMIS, a numeric attribute.

\begin{tabular}{|c|c|c|c|}
\hline Input Variables & Description of input variables & $\begin{array}{c}\text { Type of } \\
\text { variables }\end{array}$ & $\begin{array}{l}\text { Composite } \\
\text { Variables }\end{array}$ \\
\hline GENDER & Recipient Gender & Nominal & \multirow{18}{*}{ Recipient } \\
\hline $\mathrm{ABO}$ & $\begin{array}{l}\text { Recipient Blood } \\
\text { Group }\end{array}$ & Nominal & \\
\hline $\begin{array}{l}\text { TOT_-_SERUM.-- } \\
\text { ALBUM }\end{array}$ & Total Serum Albumin o recipient & Numeric & \\
\hline RESIST.. INF & $\begin{array}{l}\text { PAN Resistant Bacterial } \\
\text { Infection }\end{array}$ & Nominal & \\
\hline INIT_.. AGE & Age at time of listing & Numeric & \\
\hline ECMO_.TRR & Recipient on Life support & Numeric & \\
\hline CREAT_.TRR & $\begin{array}{l}\text { Recipient Serum Creatinine at } \\
\text { time of Transplantation }\end{array}$ & Numeric & \\
\hline $\begin{array}{l}\text { HEMO_PA MN.. } \\
\text { TRR }\end{array}$ & Current Hemodynamic PA mean & Numeric & \\
\hline IABP--TRR & $\begin{array}{l}\text { Patient on Life support at } \\
\text { Transplant }\end{array}$ & Numeric & \\
\hline ACUTE_REJ_EPI & $\begin{array}{l}\text { Did Recipient have any acute } \\
\text { rejection episode }\end{array}$ & Numeric & \\
\hline GTIME & Graft lifespan days & Numeric & \\
\hline TR_REJ1Y & $\begin{array}{l}\text { Treated for Rejection within one } \\
\text { year }\end{array}$ & Nominal & \\
\hline PRAMR_CL2 & Recipient most recent PRA\% & Numeric & \\
\hline AGE & Recipient age & Numeric & \\
\hline ISCHITIME & Ischemic time in hours & Numeric & \\
\hline $\begin{array}{l}\text { GRF_FAIL } \\
\text { CAUSE }\end{array}$ & Cause of graft failure & Nominal & \\
\hline GRF_-STATUS & Graft Status & Numeric & \\
\hline AGE__GROUPS & Recipient Age groups & Numeric & \\
\hline AGE_.DON & Donor Age & Numeric & \multirow{4}{*}{ Donor } \\
\hline ABO_.DON & Donor blood group & Nominal & \\
\hline GENDER_DON & Gender of the donor & Nominal & \\
\hline $\begin{array}{l}\text { DOPAMINE } \\
\text { DON__OLD }\end{array}$ & $\begin{array}{l}\text { Deceased donor Dopamine } \\
\text { within } 24 \text { hours Pre-cross clamp }\end{array}$ & Nominal & \\
\hline
\end{tabular}




\begin{tabular}{|c|l|l|c|}
\hline $\begin{array}{c}\text { INOTROP.- } \\
\text { AGENTS }\end{array}$ & $\begin{array}{l}\text { Deceased donor Inotrope Agents } \\
\text { at time of inclusion }\end{array}$ & Nominal & \\
\hline HLAMIS & HLA Mismatch level & Numeric & Transplantati \\
on
\end{tabular}

Table 1: Description of input parameters, characteristics with their composite variables

Table 1 comprises the elements of Input determinant of Donor, Recipient and transplantation, trait and their composite variables. The dataset consisted of donor, recipient and transplantation attributes. In order to forecast the short-term survival after Thoracic transplantation, opting the relevant input parameters is very crucial. A total of 543 attributes includes clinical and nonclinical multi-organ data. As in every dataset, some attributes could be eliminated without the help of any data mining techniques. The relevant data for Thoracic transplantation only were extracted and others were removed. The proposed study considered only the survival prediction of adult patients. Therefore the dataset consisting of 9373 records of pediatric patients were removed. For survival prediction, we considered the attributes of thoracic patients at transplantation time. Finally, through different stages of data validation, 24 important attributes with 485 records were extracted, out of which one attribute, GRF_STAT was kept as output attribute. The attributes regarding donor, recipient and transplantation are included in Table 1.

Table 2. Ranking of Input Attributes

\begin{tabular}{|c|c|c|}
\hline S1 No & Rank & Attributes \\
\hline 1 & 1 & GENDER \\
\hline 2 & 2 & ABO \\
\hline 3 & 3 & TOT_SERUM_ALBUM \\
\hline 4 & 4 & RESIST_INF \\
\hline 5 & 5 & INIT_AGE \\
\hline 6 & 6 & ECMO_TRR \\
\hline 7 & 7 & CREAT_TRR \\
\hline 8 & 8 & HEMO_PA_MN_TRR \\
\hline 9 & 9 & IABP_TRR \\
\hline 10 & 10 & ACUTE_REJ_EPI \\
\hline 11 & 11 & GTIME \\
\hline 12 & 12 & TRTREJ1Y \\
\hline 13 & 13 & HLAMIS \\
\hline 14 & 14 & PRAMR_CL2 \\
\hline 15 & 15 & AGE_DON \\
\hline 16 & 16 & ABO_DON \\
\hline 17 & 17 & GENDER_DON \\
\hline 18 & 18 & DOPAMINE_DON_OLD \\
\hline
\end{tabular}




\begin{tabular}{|c|c|c|}
\hline 19 & 19 & ABO_MAT \\
\hline 20 & 20 & AGE \\
\hline 21 & 21 & ISCHITIME \\
\hline 22 & 22 & GRF_FAIL_CAUSE \\
\hline 23 & 23 & AGE_GROUP \\
\hline 24 & 24 & INOTROP_AGENTS \\
\hline
\end{tabular}

The Table 2 shows the ranking of input attributes. The selection of attributes was conducted with the help of InfoGain Attribute Eval using the ranker search method that ranks the attributes according to their relevance. The 24 most relevant attributes were obtained which are very beneficial for the short term prediction of survival after Thoracic transplantation. Info Gain Attribute Eval assesses the value of an attribute by calculating the information gain with respect to the class.

InfoGain $($ Class, Attribute $)=H($ Class $)-H($ Class - Attribute $)$

in which $\mathrm{H}$ is the information entropy. It is the procedure by which each attribute in our data file is assessed in the context of the output variable (such as the class). The ranker search method traverse different combinations of attributes in the dataset in order to ensue on a short list of selected features. The dataset consisted of 24 clinical input attributes that help to achieve the survival prediction with increased accuracy. Age of recipient and also donor is given by AGE and AGE_DON. The gender of the recipient and donor are also represented by GENDER and GENDER_DON respectively. ABO_MAT represents the donor and recipient match level. ECMO_TRR, TOT_SERUM_ABUM, RESIST_INF, CREAT_TRR, which is the recipient's serum creatinine at the time of transplantation. HEMO_PA_MN_TRR, IABP_TRR, ACUTE_REJ_EPI, GTIME, TRTREJ1Y, PRAMR_CL2, AGE, ISCHITIME, GRF_FAIL_CAUSE are all clinical parameters of the recipient which include both numerical and nominal data types. AGE DON is the clinical numerical parameter of the donor. DOPAMINE_DON OLD, INOTROP_AGENTS, GENDER_DON, and ABO_DON are clinical parameters of the donor that are represented as nominal attributes. AGE_DON is the clinical numerical parameter of the donor. DOPAMINE_DON OLD, INOTROP_AGENTS, GENDER_DON, ABO_DON are the clinical parameters of the donor that are represented as nominal attributes. HLAMIS and ABO_MAT are the transplantation data, which are represented as 
numeric data. The GRF_STAT is taken as the output class and is not included in the input attribute description table. According to InfoGainAttributeEval, GENDER holds the highest ranking in the dataset. ABO shows the next highest ranking, TOT_SERUM_ALBUM follows and so on. In the dataset, INOTROP_AGENTS shows the lowest ranking. It is a relevant attribute for predicting survival in thoracic transplantation.

\subsection{Model Selection}

The model selected for short-term Thoracic transplantation survival prediction was the Artificial Neural Network (ANN). The proposed study consisted of multi-layer perceptron ANN Model and Radial Basis Function ANN model.

\subsubsection{Artificial Neural Network}

The first step towards an ANN modeled easy neural network with electrical circuits [2]. ANNs or connectionist systems are computing systems that are mimic but not are not similar to biological neural networks that constitute animal brains [2]. Such systems learn to perform tasks by considering examples, generally without being programmed with task specific rules. The Fig. 1. depicts the basic structure of ANN. An ANN is a data processing nonlinear model based on the neural configuration of the brain that is capable of assimilating tasks such as classification, prediction, decision-making, visualization, and other variants just by considering examples. The input layer involves input neurons that transmit information to the hidden layer [2]. The hidden layer transmits data to the output layer. Every neuron has weighted inputs (synapses), which is an activation function that defines the output when given an input and one output.

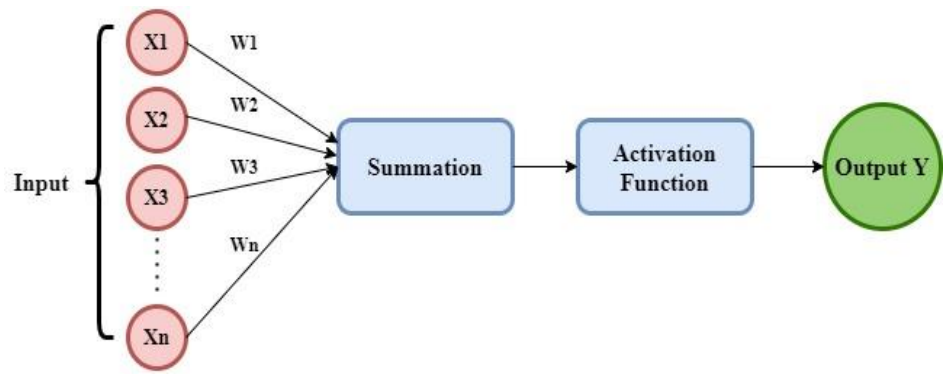

Fig. 1. Basic Structure of an artificial neural network, ANN

The Fig. 1.depicts the basic structure of ANN. An ANN is a data processing nonlinear model based on the neural configuration of the brain that is 
capable of assimilating tasks such as classification, prediction, decision-making, visualization, and other variants just by considering examples. The input layer involves input neurons that transmit information to the hidden layer [2]. The hidden layer transmits data to the output layer. Every neuron has weighted inputs (synapses), which is an activation function that defines the output when given an input and one output. Synapses are malleable parameters that transform a neural network to a parameterized system [2]. The weighted sum of the inputs generates the activation signal that is transferred to the activation function to yield one output from the neuron [2].

\subsubsection{Multilayer Perceptron ANN}

MLP model is a class of feed forward ANNs [12]. The term MLP is employed inconclusively or else loosely to ask any feed forward ANN's otherwise it is strictly used to define networks constituted of multiple layers of perceptron. MLP is a perceptron however there is the added complexity through the advent of layers [12]. We can see three types of layers in an MLP which consist of an input, hidden and output layers [12].

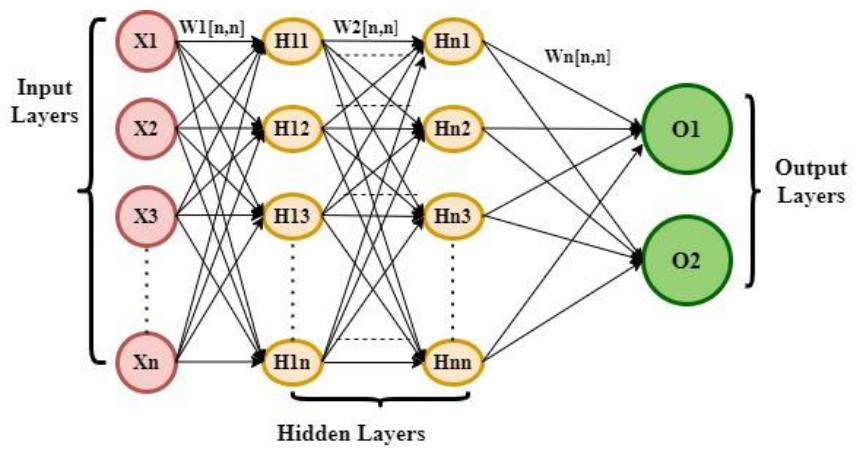

Fig. 2. Multilayer Perceptron model

MLP consists of more than one collinear layer of neurons. While considering a simple three layer perceptron, the primary layer is input layer and final is the output layer and middle layer is called as hidden layer. We grub our input file into the primary input layer and the product is taken from the output layer [12]. We will be able to raise the amount of the hidden layer as much as we wish, to build the model more composite according to our task. The feed forward network is the most neural network model [12]. The goal of the model is to estimate some function $\mathrm{f}($ ). A classifier which chart an input $\mathrm{k}$ to an output class $\mathrm{c}$ as shown as, 


$$
c=f(k)
$$

MLP spot the simplest estimation there to classifier by defining and charting,

$$
c=f(k, \theta)
$$

The outstanding parameters, theta for the classifier need to be investigated. The MLP neural networks consist of numerous functions that are grouped at once. The three province or layers of a network will form,

$$
f(k)=f(3)(f(2)(f(1)(k)))
$$

The three layers consist of components that achieve an affine alteration of a linear volume of inputs [12]. All layers are depicted as shown,

$$
c=f\left(W_{k} T+b\right)
$$

$\mathrm{n}$ which $\mathrm{f}$ is the activation function, $\mathrm{W}$ is the set of criteria or weights in the layer, $\mathrm{k}$ is the input vector, which will be the prior layer's output and $\mathrm{b}$ is that the bias vector. The MLP layers consist of distinct fully connected layers because each unit during a layer is attached to all or any other units within the preceding layer [12]. In a fully connected layer, the specification of each unit is autonomous for the remainder of the units within the layer, meaning each unit possesses a singular set of weights. Activation functions also known as irregularities describe the input or output connections in a nonlinear way. The MLP is implemented by applying the back propagation technique [12]. Fig. 2.depicts the basis structure of multi-layer perceptron model. Technically, the back-propagation algorithm is a procedure for placing the weights in a multi-layer feed-forward neural network [12]. In that scenario, it needs a network composition to be explained of further layers in which one layer is entirely attached to subsequent layer [12]. The algorithm is employed to effectually train a neural network via a principle called the chain rule [12].

\subsubsection{Back Propagation}

Back propagation is the short form of the "backward propagation of errors". It is a typical procedure for coaching ANNs. This technique helps compute the gradient of a loss function with respect to all the weights in the network [13]. The mechanism of back propagation is clearly depicted in Fig. 2. Back-propagation is the core of neural network training. It is the procedure of tweaking the weights of a neural network supporting the fault rate (for example, loss) acquired in the previous stage (for example, iteration) [13]. Appropriate 
tuning of the weights assures lower fault rates, making the model more relevant by increasing its generality [13]. The algorithm is employed to effectively instruct a neural network via the chain rule. In a back-propagation algorithm there are two passes taking place, forward and backward passes [13]. In simple terms, through a network after each forward pass, back-propagation executes a backward pass while regulating the model's parameters (weights and biases). After each forward pass, the fault is computed by evaluating the predicted output against the calculated output and back-propagates the output layer until the error vanishes [13].

\subsubsection{Radial Basis Function ANN}

A Radial Basis Function (RBF) ANN is a man-made neural network that uses an activation function called RBF [14]. The output of the network will be a consecutive amalgamation of RBFs of the input and neuronal parameters [14]. RBF network consists of three layers: an input layer, hidden layer with a nonlinear RBF as an activation function and a linear output layer.

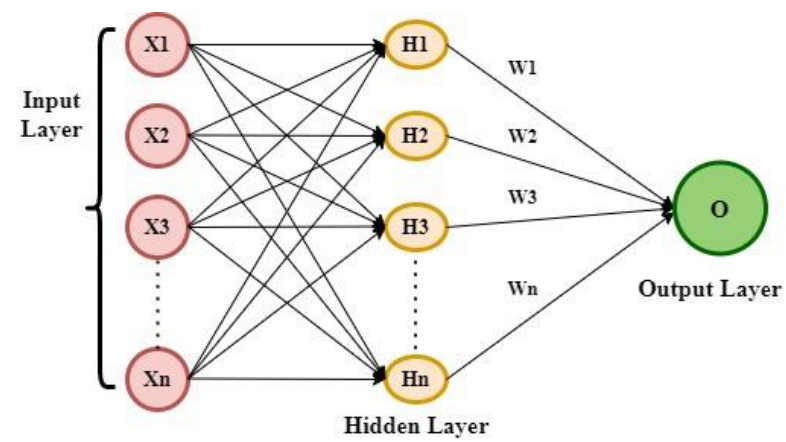

Fig. 3. Radial Basis Function model

Fig. 3.depicts the structure of a RBF network. One neuron within the input layer corresponds to every predictor variable [14]. Every neuron present in the hidden layer consists of a radial basis function (for example, Gaussian) gathered on a point with the same dimensions as the predictor variables [14]. The output layer consists of a weighted sum of outputs from the hidden layer to form the network output. RBF networks are conceptually similar to K-nearest neighbor models [14]. The basis for this model is that a predicted target value of an item is probably going to be about equivalent because the other items have close values of the predictor variables [14].

$$
\sum_{j=1}^{m} w_{j} h_{j}(x)
$$




$$
h_{j}(x)=\exp \left(-\left(x-c_{j}\right)^{2} / r_{j}^{2}\right)
$$

$\mathrm{h}(\mathrm{x})$ is the Gaussian activation function with the parameter, $\mathrm{r}$ is the radius and $\mathrm{c}$ is the center or the average taken from the input space defined individually at each RBF unit [14]. The learning process is based on adjusting the parameters of the network to propagate a set of input-output patterns.

\subsection{Model implementation}

Datasets for the study were collected from the UNOS database. It is a live multi-organ dataset, and the dataset consisted of 543 attributes and 1, 48,509 records.

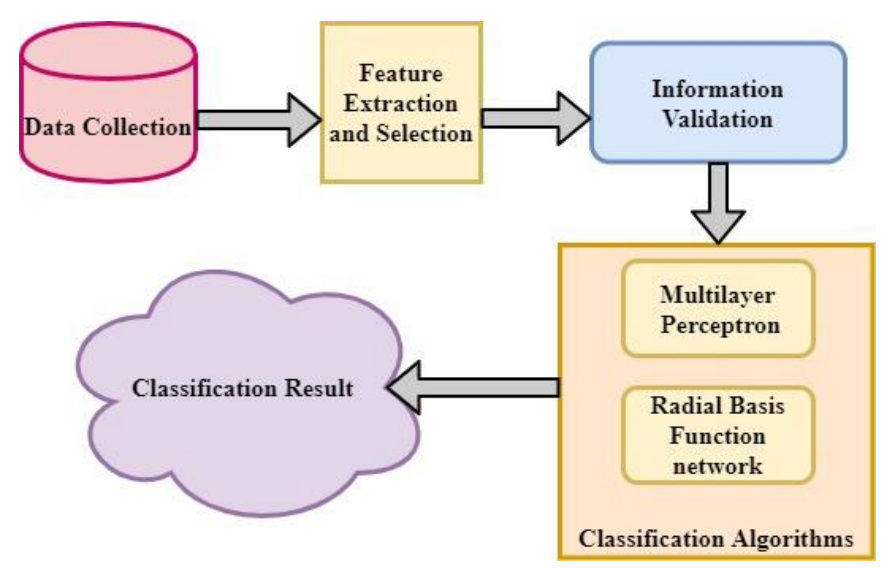

Fig. 4. System Design

Fig. 4.depicts the model implementation of the proposed study. We considered the Thoracic transplantation data only. Data validation is an important task in neural networks. Data validated and the relevant 25 attributes for the Thoracic transplantation survival prediction which includes 24 input attributes and one output attribute were extracted. The model for survival prediction was then selected which was the multi-layer perceptron. In order to prove the accuracy of the proposed model, we also implemented the RBF ANN. These 24 input attributes in the dataset were given to the MLP model and RBF network model. Thus the classification result of the system was obtained. The MLP showed a higher accuracy of survival prediction which was $97.1 \%$. In order to prove the accuracy of the proposed system, we also implemented the RBF network which achieved an accuracy of $92.37 \%$. The proposed system is a great achievement for the survival prediction of thoracic transplantation with accuracy of $97.1 \%$. 


\subsubsection{Survival Analysis Based on MLP}

The survival analysis of cardiac transplantation was calculated on the basis of number of years in the UNIOS dataset. The follow up information in the dataset was used to perform the survival analysis. Different tables were created for different year's survival prediction and linked those tables using the identifier PT_CODE. In order to do the survival analysis, the survival probabilities were calculated with the follow up information. Based upon the number of cardiac patients were alive at the start and the number of patients dead, the survival probabilities was calculated. The survival probabilities were calculated with the difference of number of patients were alive at the start and dead and was represented as SP.

$S P=(($ initial living patient's number $)-($ Died patient's number $)) /($ initial living patient's number)

The MLP model was used to train the data which consists of 4023 records of cardiac patients of three years. There were multiple records for most of the patients. Multiple datasets were trained using MLP model for cardiac survival analysis.

\subsubsection{Performance}

For the short-term prediction of survival in Thoracic transplantation, we used the ANN model and MLP and in order to prove the accuracy of the model, we trained the dataset in the RBF model also.

\subsubsection{Performance Measures}

To dictate the supreme classifier and enhance the accuracy of the model, the 10-fold cross-validation method was also used in the training set, and the training phase did not use the data from the test set. We could see that the accuracy of the two NN models were $90 \%$ among which the accuracy of the MLP was $97.1 \%$ which was the highest, followed by the RBF which was $92.37 \%$. For the performance assessment in implementation of the models, the performance measures such as TP, FP, TN and FN were symbolized as true positive (the number of actual positives), false positive (the number of actual negatives), true negative (the number of instances correctly predicted as not required) and false negative (the number of instances incorrectly predicted as not required)[15]. The 
evaluation procedures consisted of two types: (1) evaluation with performance measures and (2) evaluation with performance error measures.

$$
\begin{gathered}
\text { Accuracy }=\frac{T P+T N}{T P+F P+T N+F P} \\
\text { Precision }=\frac{T P}{T P+F P} \\
\text { Recall }=\frac{T P}{T P+F N} \\
F 1-\text { Measure }=\frac{2 \times \text { Precision } \times \text { Recall }}{\text { Precision }+ \text { Recall }}
\end{gathered}
$$

in which F1-Measure was defined as the weighted harmonic mean of the precision and recall, which depicts the inclusive performance [16]. In addition to the above mentioned evaluation benchmark, we used a receiver operating characteristic (ROC) curve and area under curve (AUC) to evaluate the assets and liabilities of the classifier [16]. The ROC curve exhibits the commutation linking the true and false positive rates (TPR and FPR) respectively [17]. If the ROC curve is adjacent to the top left corner of the graph, then the model is said to be fitter [17]. When the area of the AUC was adjacent to 1, the selected model was preferable. In medical data, more recognition is given to recall rather than accuracy [17]. When the recall rate is higher rather than lower, the chance that a patient has the threat of disease is postulated to have no disease danger.

\subsubsection{Performance Error Measures}

Performance error measures include mean absolute error, root mean square error (RMSE), relative absolute error and root relative square error (RRSE) [17]. In demography, mean absolute error (MAE) is an estimation of difference between two continuous variables. The mean absolute error is useful for expressing MAE as the sum of two constituents such as quantity disagreement and allocation disagreement. Quantity disagreement is the absolute gain of the mean error [18]. The root mean square deviation (RMSD) or RMSE is often cast off as the computation of the variation between values (sample and population values) speculated by a model or a gauge in which the values are literally declared [18]. The absolute error is the amplitude of the variation between the actual value and the approximation. The relative error is the absolute error divided by the amplitude of the exact value. The RRSE is correlative to what it would have been if an easy predictor had been utilized. Specifically, we can define simple predictor is just the median of the true values. Hence, the relative squared error considers 
the entire squared error and normalizes it by dividing the total squared error of the simple predictor [18]. By considering the square root of the relative squared error, we can reduce the fallacy of the identical measurements as the predicted consignment [18]. We can determine these measures by considering the AUC [19].

\subsection{Results and Discussion}

The dataset used for the study consisted of 485 adult records and 25 attributes. The models used in the study trained 24 input attributes and produced the survival output through the output attribute. The input attributes, state of the recipient, complications in the transplantation, and quality of the graft are the factors considered for the post transplantation outcome.

\subsubsection{Data validation in survival prediction}

In our study, 231 female recipients and 254 donor recipients waiting for donors were included. The donor data includes 184 females and 301 males. Four different blood groups such as $\mathrm{O}, \mathrm{A}, \mathrm{B}$ and $\mathrm{AB}$ for the recipients were included in the dataset. One-hundred eighty-nine recipients belonged to blood group O, twohundred eight recipients belonged to group A, sixty-one patients in group B and twenty-seven patients are in group AB. The attribute, TOT SERUM ALBUM had a maximum value of 0.8 and minimum value of 6.9 . The mean of the same attribute was represented as 3.919 and the standard deviation was 0.517 . Thirtyone records were missing in the data set. Regarding RESIST IN, four-hundred seven patients were not affected with bacterial infection. Nine patients were affected with bacterial infection and six records were represented as undefined. Three missing records were found. In the recipient age, INIT AGE, seventeen and sixty-eight were the minimum and maximum ages, respectively. This finding clearly shows that the dataset includes only adult records. The mean value of INIT AGE was 51.192 and standard deviation was 11.039. The minimum value of CREAT TRR was 0.4 and maximum value was seventeen with mean values of 0.9 and 0.784 respectively. The minimum value of HEMO PA MN TRR was eight and the maximum value was ninety nine with a mean value of 27.664 and standard deviation of 11.899. Six different blood groups such as O, A1, A, B, A2 and $\mathrm{AB}$ for the donors were found in the dataset. Two-hundred twenty-seven 
recipients belonged to blood group $\mathrm{O}$, fifty recipients belonged to group $\mathrm{A} 1$, Onehundred thirty-two patients in group A, fifty-six patients in group B, nine patients in group A2 and eleven patients in group AB. Sixty no values and Four-hundred twenty-five yes values or DOPAMINE DON OLD with two distinct values were found. The minimum age of the donors in the dataset was eighteen and maximum value was sixty eight. The minimum ischemic time was 0.9 and maximum ischemic value was twelve with a mean value of 4.651 and standard deviation of 10.943.

\subsubsection{Survival Analysis With Respect to Survival Probabilities}

While classifying the records for three years including six months, we obtained 391 records. Initially the UNOS dataset included 405 records of cardiac patients.

Table 3: Survival analysis with respect to survival probabilities

\begin{tabular}{|l|l|l|l|l|}
\hline $\begin{array}{c}\text { Patient's follow } \\
\text { up period in } \\
\text { years }\end{array}$ & 0.5 & 1 & 2 & 3 \\
\hline $\begin{array}{l}\text { No of patients } \\
\text { survived }\end{array}$ & 391 & 387 & 384 & 378 \\
\hline $\begin{array}{l}\text { Survival } \\
\text { probabilities }\end{array}$ & 96.5 & 98.9 & 99.2 & 97.6 \\
\hline
\end{tabular}

Out of 405 records, 14 patients were dead after six months. Out of 14 cardiac patients, six patients were died after one month of cardiac transplantation. Three patients were died after 2 months of transplantation. Subsequently, one patient died after three months, again one patient died after four months, two patients died after five months and one patient died after six months of cardiac transplantation. While performing the survival analysis we could observe that 387 patients were alive after one year, 384 patients after 2 years and 378 patients after 3 years of cardiac transplantation. The survival analysis with respect to survival probabilities is listed in Table 3.

\subsubsection{Performance Evaluation of the proposed models}

The Table 4 depicts the performance measures of the proposed classifiers, MLP and RBF. The accuracy of the proposed MLP was very high, 97.1\%. The 
RBF model had an accuracy of $92.37 \%$. Sensitivity and specificity of the proposed MLP were 0.966 and 0.984 respectively and the precision value was 0.972. Sensitivity and specificity values of RBF were 0.935 and 0.893 respectively. RBF had a precision of 0.923 . The results of recall and F-measure obtained from the MLP model were 0.971 and 0.971 . TP and FP rates of the RBF were 0.924 and 0.129 respectively. Although the time taken for training the dataset in RBF was less than for the MLP, the performance in terms of accuracy was more in MLP than in the RBF.

Table 4. Performance measures of MLP and RBF

\begin{tabular}{|c|c|c|c|c|c|c|c|c|c|}
\hline $\begin{array}{c}\text { Rerformance } \\
\text { Measures }\end{array}$ & $\begin{array}{c}\text { Sensi } \\
\text { tivity }\end{array}$ & $\begin{array}{c}\text { Specificit } \\
\mathrm{y}\end{array}$ & $\begin{array}{c}\text { Accur } \\
\text { acy }\end{array}$ & Precision & Recall & $\begin{array}{c}\text { F- } \\
\text { Meas } \\
\text { ure }\end{array}$ & $\begin{array}{c}\text { ROC } \\
\text { Area }\end{array}$ & $\begin{array}{c}\text { TP } \\
\text { Rate }\end{array}$ & $\begin{array}{c}\text { FP } \\
\text { Rate }\end{array}$ \\
\hline MLP & 0.966 & 0.984 & $\begin{array}{c}97.113 \\
\%\end{array}$ & 0.972 & 0.971 & 0.971 & 0.918 & 0.971 & 0.086 \\
\hline RBF & 0.935 & 0.893 & $\begin{array}{c}92.37 \\
\%\end{array}$ & 0.923 & 0.924 & 0.923 & 0.95 & 0.924 & 0.129 \\
\hline
\end{tabular}

ROC Area: Receiver Operating Characteristics Area, TP Rate: Total Positive Rate, FP Rate: False Positive Rate

Table 5. depicts the performance error measures of MLP and RBF model. From Table 4, it can be seen that MAE of the MLP was only 0.0309 and that of the RMSE was0.165. The RAE of MLP was only $7.515 \%$ and RRSE was only $36.423 \%$. The MAE value of RBF was 0.1149 and RMSE was 0.2501 . The RAE value of RBF was $27.944 \%$ and that of the RRSE value was $55.198 \%$.

Table 5. Performance Error measures of MLP and RBF

\begin{tabular}{|c|c|c|c|c|}
\hline $\begin{array}{c}\text { Performance } \\
\text { Elassifier } \\
\text { Measures }\end{array}$ & MAE & RMSE & RAE & RRSE \\
\hline MLP & 0.0309 & 0.165 & $7.515 \%$ & $36.423 \%$ \\
\hline RBF & 0.1149 & 0.2501 & $27.944 \%$ & $55.198 \%$ \\
\hline
\end{tabular}

MAE: Mean Absolute Error, RMSE: Root Mean Squared Error, RAE: Relative Absolute Error, RRSE: Root Relative Squared Error

\subsection{Analysis of Results}

The outcome of the proposed MLP and RBF ANN models for the short term survival prediction of Thoracic transplantation were implemented and 
evaluated in terms of performance measures and performance error measures. The output survival prognosis was determined using these computed values and depicted the survival output was depicted using ROC curve. The confusion matrix of MLP model is more precise than that of the RBF model. The correctly classified instances of MLP included 471 and incorrectly classified instances included 14. In the case of RBF, correctly classified instances included 448 and incorrectly classified instances included 37 out of the total 485 instances. The FP Rate of MLP was 0.086 and that of RBF was 0.129 . Here we can see that classification is more accurate for MLP model than RBF.

\subsubsection{Proposed Model Comparison with respect to Performance Measures}

The performance comparison of RBF and MLP ANN models are depicted in Table 4 and 5. The accuracy was higher for the MLP model than for the RBF. MLP has an accuracy of $97.1 \%$ while RBF model has an accuracy of $92.37 \%$. The TP Rate was high for MLP with 0.971 while RBF was 0.924. In order to demonstrate the accuracy of proposed MLP classifier we also implemented the RBF which clearly shows that MLP with back-propagation had a higher accurate survival prediction of thoracic transplantation than that of the RBF. From the comparison, it is very clear that MLP had a higher AUC than that of RBF that shows a higher accurate classification of MLP model. Even though the RBF takes less time to build than that of MLP, the accuracy is less than MLP.

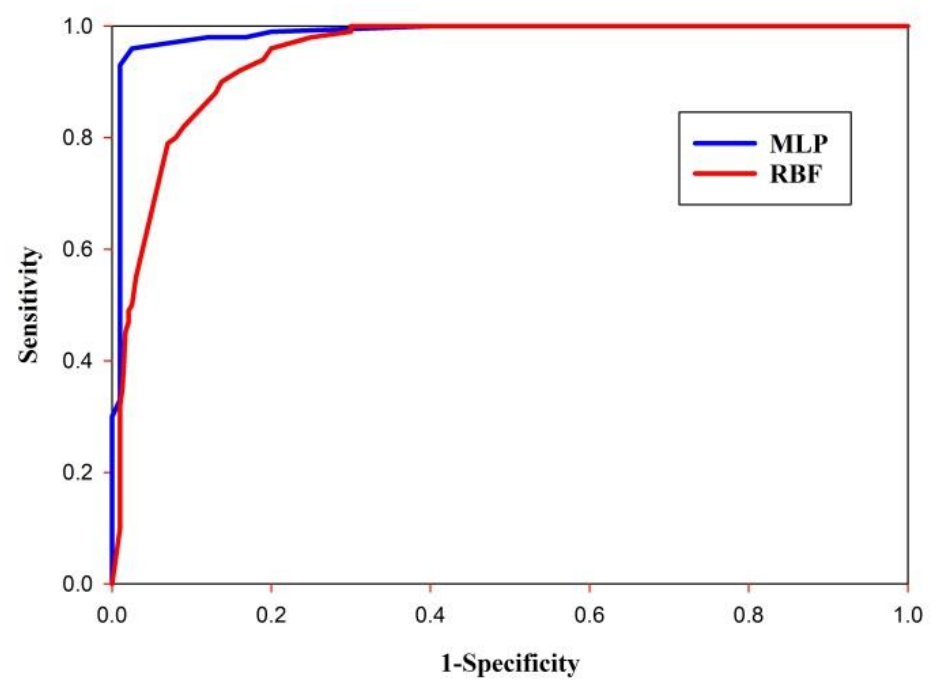

Fig. 5. Comparison between classifiers MLP and RBF 
Studies have shown that the models chosen for the classification purpose contain an AUC value 0.5. Thus, MLP and RBF models can be selected for medical purposes with a higher priority to MLP. Also Fig. 5 depicts the ROC curve of the proposed MLP classifier. The ROC area of the proposed MLP was 0.918. The ROC curve was graphed with sensitivity on the Y-axis and 1specificity on the X-axis. The TP rate from the RBF model was 0.924 and FP rate was 0.129 . The ROC area results from the RBF model was 0.95. The MAE was 0.0309 for MLP and 0.1149 for RBF. RAE was $7.515 \%$ for MLP and $27.944 \%$ for RBF. The RRSE was $36.423 \%$ for MLP and $55.198 \%$ for RBF. We can see that the error rate was higher for RBF than MLP. Hence, the MLP with backpropagation had a high accurate survival prediction than that of RBF.

\subsubsection{Comparison Of the proposed system with existing system}

Fig. 6. compared the proposed system with the existing system. The existing system that we considered was predicting the graft survival of heart lung transplant patients [4]. The existing system we used was from the research work of Ostekin in 2009 [4]. The system aimed to forecast the integrated survival of heart lung transplant patients. The system used a different dataset than the proposed system and was implemented with MLP, decision tree and logistic regression [4] out of which the MLP produced the higher accuracy of 85.9\%. Our proposed system also implemented MLP with a dataset collected from UNOS and extracted the relevant attributes for the survival prediction. The system implemented MLP with back-propagation and obtained a higher accuracy of 97.1\%. Again, in the Fig. 6. we compared our classifier result with the existing MLP model having different attributes. It is very clear that the accuracy of the proposed MLP classifier was elevated compared to that of existing system. Table 6 depicts the performance differentiation of proposed system with the existing system. Sensitivity of the existing system was 0.847 and sensitivity of proposed system was 0.966 . Specificity of the existing system was 0.869 and the specificity of the proposed system was 0.984 . We can also see the difference in the accuracy which is $97.1 \%$ and $85.9 \%$ for the proposed and existing systems, respectively [4]. In short, our proposed system for survival prediction of Thoracic transplantation has a higher accuracy of $97.1 \%$, which was obtained by implementing our relevant dataset in MLP with back-propagation. Hence, the proposed system had a higher survival prediction than the existing systems. 
Table 6. Performance Comparison of Proposed System with Existing System

\begin{tabular}{|c|c|c|}
\hline Performance measures & Proposed MLP & Existing MLP \\
\hline Accuracy & $97.113 \%$ & $85.9 \%$ \\
\hline Sensitivity & 0.966 & 0.847 \\
\hline Specificity & 0.984 & 0.867 \\
\hline
\end{tabular}

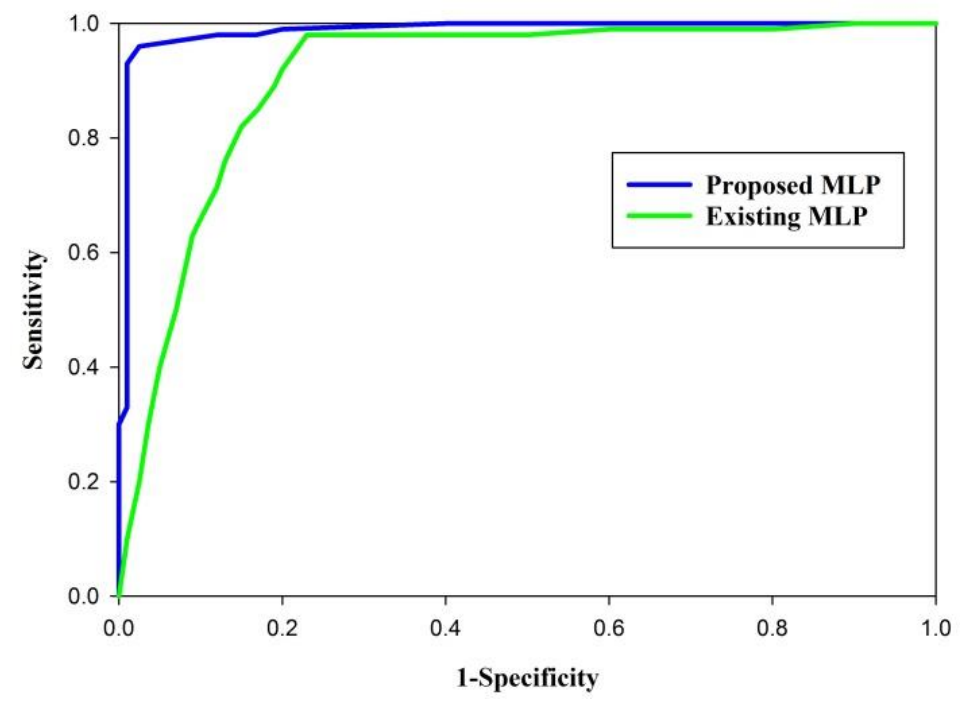

Fig. 6. Performance analysis of proposed system with existing system

\section{Conclusion}

Thoracic transplantation and the survival after the thoracic transplantation is a hot area of research. The donor scarcity is an important problem and hence in such a synopsis, every organ allocation has to be accurate. We all know that survival from Thoracic transplantation is very risky in the medical domain. Hence, we propose a computational model for survival prediction for which we used a relevant live dataset. Data were validated accurately and 25 relevant attributes for survival prediction were extracted and our dataset was implemented in the selected MLP model with back-propagation. The proposed system obtained a higher accuracy of $97.1 \%$. In order to forecast the accuracy of proposed model, we implemented another computational model RBF. It yielded an accuracy of $92.37 \%$, which was less than that of the proposed model. As it is a life problem and in order to prove the accuracy of model, we compared the proposed system with an existing system. The existing system branched off the MLP model and was used to forecast the survival of heart lung transplant patients with another dataset, which produced an accuracy of only $85.9 \%$. Hence, through all of these comparisons, we came to the conclusion that our proposed model with our 
relevant dataset had a higher accuracy of survival prediction in thoracic transplantation than the existing systems. The results will be very supportable for doctors for undertaking lifesaving procedures for the patients.

\section{Abbreviations}

TT: Thoracic transplantation, ANN: Artificial Neural Networks, UNOS: United Network for Organ Sharing, IMPACT: Index for Mortality Prediction After Cardiac Transplantation, OPTN: Organ Procurement and Transplant Network, MLP: Multi-layer Perceptron, RBF: Radial Basis Function, ROC: Receiver Operating Characteristic, AUC: Area Under Curve, RMSE: Root Mean Square Error, MAE: Mean Absolute Error, RRSE: Root Relative Square Error.

\section{Declarations}

\section{Ethics Approval and Consent to participate}

The data used for this study was collected based on Organ Procurement and Transplantation Network data as of 5th June 2015. This research work was supported in part by Health Resources and Services Administration contract 2342005-370011C. The content is the responsibility of the authors alone and does not necessarily reflect the views or policies of the Department of Health and Human Services, nor does mention of trade names, commercial products, or organizations approved by the U.S. Government.

\section{Consent for publication}

Not applicable.

\section{Availability of data and materials}

Not applicable.

\section{Competing interests}

The authors declare that they do have no competing interests available.

\section{Funding}

Not applicable.

\section{Authors' contributions}

This study was designed and compiled by RCG as the principal investigator. The development of the basic research questions, identifying the problems and selecting appropriate statistical models, data collection, data analysis, interpretation, and critical review of the paper have been done by RCG and SAK. 
The edition of the overall progress of the work was supported by RCG and SAK. All authors read and approved the final manuscript.

\section{Acknowledgements}

Not applicable.

\section{Author details}

${ }^{1, *, 2}$ Department of Computer Science and Engineering, MEA Engineering College, Perinthalmanna, Kerala, India,

\section{References}

[1] Agrawal, Ankit, et al. "Heart transplant outcome prediction using unos data." Proceedings of the KDD Workshop on Data Mining for Healthcare (DMH). 2013.

[2] Zupan, Jure. "Basics of artificial neural network." Nature-inspired Methods in Chemometrics: Genetic Algorithms and Artificial Neural Networks (Leardi, R., ed.) (2003): 199-229.

[3] Davies, Ryan R., et al. "Predicting survival among high-risk pediatric cardiac transplant recipients: an analysis of the United Network for Organ Sharing database.” The Journal of thoracic and cardiovascular surgery135.1 (2008): 147-155.

[4] Oztekin, Asil, Dursun Delen, and Zhenyu James Kong. "Predicting the graft survival for heartlung transplantation patients: An integrated data mining methodology." International journal of medical informatics 78.12 (2009): e84-e96.

[5] Weiss, Eric S., et al.'Development of a quantitative donor risk index to predict short-term mortality in orthotropic heart transplantation." The Journal of heart and lung transplantation 31.3 (2012): 266-273.

[6] Urban, Marian, et al. "Donor and recipient risk factor analysis of inferior post heart transplantation outcome in the era of durable mechanical assist devices." Clinical transplantation 32.10 (2018): e13390.

[7] Chokshi, Aalap, et al. "Hepatic dysfunction and survival after orthotropic heart transplantation: application of the MELD scoring system for outcome prediction.” The Journal of heart and lung transplantation 31.6 (2012): 591-600.

[8] Medved, Dennis, Pierre Nugues, and Johan Nilsson. "Selection of an optimal feature set to predict heart transplantation outcomes." 2016 38th Annual International Conference of the IEEE Engineering in Medicine and Biology Society (EMBC). IEEE, 2016.

[9] Dag, Ali, et al. "Predicting heart transplantation outcomes through data analytics." Decision Support Systems 94 (2017): 42-52.

[10] Tong, Li, et al. "Predicting heart rejection using histopathological whole-slide imaging and deep neural network with dropout.” 2017 IEEE EMBS International Conference on Biomedical Health Informatics (BHI). IEEE, 2017.

[11] Raji C. G. and SS Vinod Chandra. "Computer based prognosis model with dimensionality reduction and validation of attributes for prolonged survival prediction." Informatics in Medicine Unlocked 9 (2017): 93-106. 
[12] Raji C. G. and SS Vinod Chandra. "Long-term forecasting the survival in liver transplantation using multilayer perceptron networks." IEEE Transactions on Systems, Man, and Cybernetics: Systems 47.8 (2017): 2318-2329.

[13] Mozolin, Mikhail, J-C. Thill and E. Lynn Usery. "Trip distribution forecasting with multilayer perceptron neural networks: A critical evaluation." Transportation Research Part B: Methodological 34.1 (2000): 53-73.

[14] Siregar, Sandy Putra, and Anjar Wanto. "Analysis of Artificial Neural Network Accuracy Using Back propagation Algorithm In Predicting Process (Forecasting)." IJISTECH (International Journal of Information System Technology) 1.1 (2017): 34-42.

[15] Kelwade, Jairam P., and Suresh S. Salankar. "Prediction of heart abnormalities using particle swarm optimization in radial basis function neural network." 2016 international conference on automatic control and dynamic optimization techniques (ICACDOT). IEEE, 2016.

[16] Min Chen, Yixue Hao, Kai Hwang, Lu Wang, Lin Wang. "Disease Prediction by Machine Learning Over Big Data From Healthcare Communities", IEEE Access, 2017.

[17] Raji C. G., and SS Vinod Chandra. "Artificial neural networks in prediction of patient survival after liver transplantation.” J. Health. Med. Inform 7.1 (2016).

[18] Raji C. G., and SS Vinod Chandra. "Graft survival prediction in liver transplantation using artificial neural network models." Journal of computational science 16 (2016): 72-78.

[19] Raji C. G. and SS Vinod Chandra. S.S, Predicting the survival of graft following liver transplantation using a nonlinear model, Journal of Public Health, Springer, 24.5, 443-452. May 2016.

[20] Su Zhang, Xinming Ou, Doina Caragea. "Predicting Cyber Risks through National Vulnerability Database", Information Security Journal: A Global Perspective, 2015. 